\title{
Análise de frutos e nozes de cultivares de nogueira-macadâmia
}

\author{
Analysis of fruits and nuts of macadamia walnut cultivars \\ Edwaldo dos Santos Penoni ${ }^{I}$ Rafael Pio ${ }^{I *}$ Filipe Almendagna Rodrigues \\ Luana Aparecida Castilho Maro ${ }^{I}$ Fernanda Carvalho Costa ${ }^{\mathrm{I}}$
}

- NOTA -

RESUMO

A produção da nogueira-macadâmia é quantificada em "noz em casca" (casca e amêndoa). Assim, cultivares que possuam maior calibre detêm maior valor comercial. Sendo assim, o objetivo do presente trabalho foi realizar a análise de frutos e nozes de 22 cultivares de nogueiramacadâmia. As cultivares utilizados foram: 'África', 'Beaumont' (695), 'Cannon', 'C160', 'Doroti', 'Edson', 'Flor Rosa', 'HAES 722', 'HAES 814', 'HAES 816', 'HAES 842', 'HAES 849', 'IAC Campinas-B', 'IAC 9-20', 'IAC 9-20X', 'IAC 4-12B', 'Kau' ('HAES 344'), 'Keauhou' ('HAES 246'), 'Keaumi' ('IAC 4-20'), 'Mauka' ('HAES 741'), 'Pahala' ('HAES 788') e '791 Fuji'. Foram determinadas as dimensões (comprimento e diâmetro) e a massa fresca do carpelo, casca e amêndoa, além da massa fresca da "noz em casca" (casca e amêndoa), do TR (relação entre a massa das nozes em casca e a massa das amêndoas resgatadas). As cultivares apresentam diferenças quanto as dimensões dos frutos. A cultivar 'Edson' apresentou frutos e nozes com maiores dimensões e massa. Apenas as cultivares 'Pahala', 'Beaumont', 'IAC 9-20X' $e$ 'Keaumi' apresentaram TR menor que 30\%.

Palavras-chave: Macadamia integrifolia, caracterização física, amêndoa, noz em casca, TR.

\section{ABSTRACT}

The production of the macadamia walnut is measured in "nut in peel" (peel and almond). The cultivar that have higher caliber has greater commercial value. The objective of the present research was to analysis of fruits and nuts of 22 cultivars of macadamia walnut. The cultivars used were: 'África', 'Beaumont' (695), 'Cannon', 'C160', 'Doroti', 'Edson', 'Flor Rosa', 'HAES 722', 'HAES 814', 'HAES 816', 'HAES 842', 'HAES 849', 'IAC Campinas-B', 'IAC 9-20', 'IAC 9-20X', 'IAC 4-12B', 'Kau' ('HAES 344'), 'Keauhou'
('HAES 246'), 'Keaumi' ('IAC 4-20'), 'Mauka' ('HAES 741'), 'Pahala' ('HAES 788') and '791 Fuji'. It was determined the dimensions (length and diameter) and the fresh weight of the carpelo, peel and almond, besides the fresh weight of the "nut in peel" (peel and almond), besides TR (relationship among the weight of the nut in peel and the weight of the rescued almonds). The cultivars presented differences as the dimensions of the fruits. The cultivar 'Edson' presented fruits and nuts with larger dimensions and weight. The cultivars 'Pahala', 'Beaumont', 'IAC 9-20X' and 'Keaumi' presented TR lower than $30 \%$.

Key words: Macadamia integrifolia, physical characterization, almond, nut in peel, TR.

A nogueira-macadâmia (Macadamia integrifolia Maiden \& Betche) produz uma noz de alto valor no mercado internacional, com grande aceitação entres os consumidores. Nativa da Austrália, ao ser introduzida na Califórnia e Havaí foram iniciados os trabalhos de seleção varietal. Do programa de melhoramento genético do Havaí, foram lançadas as cultivares de nogueira-macadâmia da série HAES, como 'Pahala' ('HAES 788'), 'Kau' ('HAES 344'), 'Keauhou' ('HAES 246'), 'Mauka' ('HAES 741'), 'Ikaika' ('HAES 333'), 'Kakea' ('HAES 508'), 'Keaau' ('HAES 660'), 'Makai' ('HAES 800'), 'Keauhou' ('HAES 224'), dentre outras de menor importância comercial (PEACE et al., 2005). No Brasil, os trabalhos de melhoramento genético com a nogueira-macadâmia iniciaram-se no IAC, que resultou no lançamento de diversas seleções para

'Departamento de Agricultura, Universidade Federal de Lavras (UFLA, 37200-000, Lavras, MG, Brasil. E-mail: rafaelpio@dag.ufla.br. *Autor para correspondência. 
plantios comerciais (SOBIERAJSKI et al., 2006). Apesar da grande quantidade de cultivares disponíveis no Estado de São Paulo, maior produtor nacional, apenas são cultivadas 'Keauhou', 'Mauka', 'IAC CampinasB' e 'IAC 9-20' (BARBOSA et al., 2003). Acredita-se que a baixa diversificação de cultivares possa estar atrelada ao fato de a exploração da nogueira-macadâmia ser recente no Brasil e as informações sobre as tecnologias de cultivo estarem baseadas nos resultados de pesquisa da Austrália e Havaí.

A produção da nogueira-macadâmia é quantificada em "noz em casca" (casca e amêndoa) (SOBIERAJSKI et al., 2006). Sendo assim, a análise dos frutos em diferentes cultivares de nogueira-macadâmia poderia fornecer informações fundamentais para a exploração de cultivares produtoras de "noz em casca" superiores e com amêndoas de maior calibre. A partir da análise do calibre e massa, pode-se calcular e correlacionar o rendimento de polpa e nozes dos frutos, índice importante tanto para o consumo ao natural, como para a utilização agroindustrial (CARVALHO et al., 2003).

Não obstante as características intrínsecas dos frutos é considerado como principal indicador para a nogueira-macadâmia o TR (relação entre a massa da "noz em casca" e a massa da amêndoa resgatada). Algumas cultivares podem apresentar baixa produtividade, mas com alto valor de TR, o qual define o preço da noz no mercado, compensando, nesse caso, o investimento nesses materiais genéticos quando se pensa em processamento agroindustrial de noz inteira condimentada. No Brasil, o TR varia ao redor de $23 \%$ para as principais cultivares em São Paulo, porém, o valor pago pelas agroindústrias processadoras segue uma escala exponencial em função do seu aumento.

Visando a detectar nogueiras-macadâmia produtoras de frutos e nozes superiores, o presente trabalho teve como objetivo realizar a análise do calibre e massa em frutos e nozes em diferentes cultivares.

Foram coletados frutos inteiros (carpelo, casca e amêndoa - noz) no mês de março de 2011, em plantas de cinco anos de idade, quando estas estavam em plena produção. Para isso, foram identificadas aleatoriamente quatro plantas de 22 cultivares de nogueira-macadâmia, nas dependências da Macadâmia Brasilis LTDA., localizada no município de Itapira-SP. As cultivares de nogueira-macadâmia escolhidas foram: 'África', 'Beaumont' (695), 'Cannon', 'C160', 'Doroti', 'Edson', 'Flor Rosa', 'HAES 722', 'HAES 814', 'HAES 816', 'HAES 842', 'HAES 849', 'IAC Campinas-B', 'IAC 9-20', 'IAC 9-20X', 'IAC 4-12B', 'Kau' ('HAES 344'), 'Keauhou' ('HAES 246'), 'Keaumi' ('IAC 4-20'), 'Mauka' ('HAES 741'), 'Pahala' ('HAES 788') e '791
Fuji'. De cada planta, foram coletados 120 frutos, que foram homogeneizados em um recipiente e deste foram resgatados 100 frutos, que foram divididos em quatro repetições contendo 25 frutos cada, constituindo assim a parcela. $\mathrm{O}$ delineamento adotado foi o inteiramente casualizado. Os frutos foram acondicionados em sacos plásticos devidamente identificados e instantaneamente iniciaram-se as avaliações. Com o auxílio de paquímetro digital e balança de precisão, foram determinadas as dimensões (comprimento e diâmetro - face longitudinal, $90^{\circ}$ do orifício da casca) e a massa fresca do carpelo, casca e amêndoa, além da massa fresca da "noz em casca" (casca e amêndoa). Posteriormente, foi calculado o TR, expresso em porcentagem. Os dados foram submetidos à análise de variância e as médias comparadas pelo teste ScottKnott, ao nível de 5\% de probabilidade, de acordo com as indicações de GOMES (2000), utilizando-se o Sistema para Análise de Variância - SISVAR (FERREIRA, 2000).

De acordo com a análise estatística, houve diferença entre as médias em todas as variáveis mensuradas. A cultivar 'Edson' foi a que apresentou maior dimensão do carpelo $(38,91 \mathrm{~mm}$ de comprimento e 33,6mm de diâmetro) (Tabela 1) e a maior massa carpelar $(6,18 \mathrm{~g})$ (Tabela 2). Apesar de o carpelo ser destacado da "noz em casca", através do descarpelador nas unidades de beneficiamento (SOBIERAJSKI et al., 2006), cultivares com maior massa carpelar resultariam em quantidades superiores de resíduos a serem utilizados na ciclagem de nutrientes para as nogueirasmacadâmia, sendo posteriormente triturados e podendo ser depositado sobre o solo.

Quanto às dimensões da casca, a nogueiramacadâmia 'Edson' apresentou maiores dimensões e massa, não diferindo da cultivar 'Cannon', que apresentou maiores valores do diâmetro e massa (Tabelas 1 e 2). Já para a amêndoa, as cultivares que apresentaram comprimentos superiores foram 'Edson' e 'IAC 4-12B', enquanto 'Edson', 'Kau', 'Cannon', 'C160', 'IAC 4-12B' e 'HAES 816' apresentaram maior diâmetro (Tabela 1). Por outro lado, as maiores massas médias das amêndoas foram registradas para as cultivares 'Edson', 'Cannon', 'C160', 'HAES 816', 'Doroti' e '791 Fuji' (Tabela 2).

Ressalta-se que as cultivares 'Doroti' e '791 Fuji' apresentaram elevada massa fresca da amêndoa, porém não apresentaram destaque quanto às dimensões da amêndoa, em relação às demais cultivares analisadas. Por outro lado, a cultivar 'IAC 4-12B' se destacou quanto às dimensões da amêndoa, mas não apresentou elevada massa. Por esses resultados, salienta-se que não há possibilidade de se fazer uma relação entre as dimensões (comprimento e diâmetro) 
Tabela 1 - Dimensões dos frutos (carpelo, casca e amêndoa) de 22 cultivares de nogueira-macadâmia cultivadas na região Leste paulista. Lavras, UFLA, 2011.

\begin{tabular}{|c|c|c|c|c|c|c|}
\hline \multirow{2}{*}{ Cultivares } & \multicolumn{2}{|c|}{ Dimensões carpelo (mm) } & \multicolumn{2}{|c|}{ Dimensões casca (mm) } & \multicolumn{2}{|c|}{ Dimensões amêndoa (mm) } \\
\hline & Comprimento & Diâmetro & Comprimento & Diâmetro & Comprimento & Diâmetro \\
\hline 'Edson' & 38,91 a & $33,60 \mathrm{a}$ & $28,70 \mathrm{a}$ & $26,28 \mathrm{a}$ & $18,81 \mathrm{a}$ & 19,92 a \\
\hline 'Pahala (HAES 788)' & $35,99 \mathrm{~b}$ & $28,71 \mathrm{~d}$ & $24,85 \mathrm{c}$ & $23,62 \mathrm{c}$ & $15,18 \mathrm{c}$ & $17,84 \mathrm{~b}$ \\
\hline 'Beaumont (695)' & $34,56 \mathrm{c}$ & $31,09 \mathrm{c}$ & $24,38 \mathrm{c}$ & $23,90 \mathrm{c}$ & $13,16 \mathrm{~d}$ & $15,62 \mathrm{c}$ \\
\hline 'Flor Rosa' & $26,85 \mathrm{f}$ & $26,23 \mathrm{e}$ & $21,49 \mathrm{e}$ & $21,69 \mathrm{e}$ & $15,36 \mathrm{c}$ & $16,57 \mathrm{c}$ \\
\hline 'IAC 9-20X' & $33,43 \mathrm{c}$ & $29,81 \mathrm{c}$ & $23,74 \mathrm{~d}$ & $23,81 \mathrm{c}$ & $15,45 \mathrm{c}$ & $18,13 \mathrm{~b}$ \\
\hline 'Kau (HAES 344)’ & $30,86 \mathrm{~d}$ & $30,51 \mathrm{c}$ & $24,54 \mathrm{c}$ & $24,63 \mathrm{~b}$ & $15,91 \mathrm{c}$ & 18,87 a \\
\hline 'Cannon' & $34,30 \mathrm{c}$ & $32,14 \mathrm{~b}$ & $26,78 \mathrm{~b}$ & 27,11 a & $15,97 \mathrm{c}$ & 20,06 a \\
\hline 'IAC 9-20' & $30,62 \mathrm{~d}$ & $29,80 \mathrm{c}$ & $23,58 \mathrm{~d}$ & $24,33 \mathrm{c}$ & $15,66 \mathrm{c}$ & $18,05 \mathrm{~b}$ \\
\hline 'C160' & $33,74 \mathrm{c}$ & $31,91 \mathrm{~b}$ & $26,32 \mathrm{~b}$ & $25,46 \mathrm{~b}$ & $17,48 \mathrm{~b}$ & $20,31 \mathrm{a}$ \\
\hline 'HAES 849’ & $32,27 \mathrm{c}$ & $28,30 \mathrm{~d}$ & $23,38 \mathrm{~d}$ & $24,09 \mathrm{c}$ & $16,36 \mathrm{c}$ & $18,34 \mathrm{~b}$ \\
\hline 'IAC 4-12B' & $30,54 \mathrm{~d}$ & $27,39 \mathrm{~d}$ & $21,61 \mathrm{e}$ & $23,25 \mathrm{c}$ & 18,19 a & 20,49 a \\
\hline 'HAES 816' & $32,67 \mathrm{c}$ & $29,34 \mathrm{c}$ & $24,62 \mathrm{c}$ & $24,92 \mathrm{~b}$ & $16,58 \mathrm{~b}$ & 19,65 a \\
\hline 'Doroti' & $33,31 \mathrm{c}$ & $29,52 \mathrm{c}$ & $24,49 \mathrm{c}$ & $24,88 \mathrm{~b}$ & $14,98 \mathrm{c}$ & $18,38 \mathrm{~b}$ \\
\hline ‘791 Fuji’ & $30,47 \mathrm{~d}$ & $26,54 \mathrm{e}$ & $24,57 \mathrm{c}$ & $24,30 \mathrm{c}$ & $15,46 \mathrm{c}$ & $18,09 \mathrm{~b}$ \\
\hline 'Keaumi (IAC 4-20)' & $32,87 \mathrm{c}$ & $27,23 \mathrm{~d}$ & $23,51 \mathrm{~d}$ & $23,67 \mathrm{c}$ & $15,14 \mathrm{c}$ & $15,00 \mathrm{c}$ \\
\hline 'HAES 814' & $27,10 \mathrm{f}$ & $25,32 \mathrm{e}$ & $22,30 \mathrm{e}$ & $22,85 \mathrm{~d}$ & $14,14 \mathrm{~d}$ & $16,92 \mathrm{c}$ \\
\hline 'HAES 722' & $32,37 \mathrm{c}$ & 26,49 e & $26,14 \mathrm{~b}$ & $24,40 \mathrm{c}$ & $15,19 \mathrm{c}$ & $18,27 \mathrm{~b}$ \\
\hline 'África' & $36,32 \mathrm{~b}$ & $30,41 \mathrm{c}$ & $25,76 \mathrm{~b}$ & $25,19 \mathrm{~b}$ & $16,04 \mathrm{c}$ & $18,61 \mathrm{~b}$ \\
\hline 'IAC Campinas-B' & $28,63 \mathrm{e}$ & $24,93 \mathrm{e}$ & $23,09 \mathrm{~d}$ & $23,49 \mathrm{c}$ & $14,09 \mathrm{~d}$ & $17,08 \mathrm{c}$ \\
\hline 'Keauhou (HAES 246)' & $31,74 \mathrm{~d}$ & $28,13 \mathrm{~d}$ & $23,55 \mathrm{~d}$ & $23,89 \mathrm{c}$ & $14,78 \mathrm{c}$ & $17,34 \mathrm{c}$ \\
\hline 'Mauka (HAES 741)' & $29,61 \mathrm{~d}$ & $25,95 \mathrm{e}$ & $22,09 \mathrm{e}$ & $21,72 \mathrm{e}$ & $14,14 \mathrm{~d}$ & $16,70 \mathrm{c}$ \\
\hline 'HAES 842' & $30,62 \mathrm{~d}$ & $29,27 \mathrm{c}$ & $23,20 \mathrm{~d}$ & $23,87 \mathrm{c}$ & $15,71 \mathrm{c}$ & $18,20 \mathrm{~b}$ \\
\hline $\mathrm{CV}(\%)$ & 3,50 & 3,32 & 4,15 & 3,30 & 6,79 & 4,82 \\
\hline
\end{tabular}

*Médias não seguidas pela mesma letra diferem significativamente entre si, pelo teste Scott-Knott, em nível de 5\% de probabilidade.

com a massa fresca. Essa falta de sincronia entre dimensões e massa pode estar relacionada ao teor de umidade das amêndoas e ou à quantidade de óleo presente nas amêndoas.

Outro ponto que deve ser ressaltado é a relação da massa da casca e da amêndoa, quanto a germinação das sementes. A nogueira-macadâmia é propagada por enxertia, não há um porta-enxerto específico e eles são produzidos pelo processo seminífero (DALASTRA et al., 2010). Sendo assim, cultivares com menores dimensões da casca podem influenciar positivamente na permeabilidade de água, que por sua vez ativam os processos metabólicos relacionados à germinação, mas, por outro lado, devem ser detentores de elevada massa de amêndoa, para assim apresentarem bom desenvolvimento das plântulas. É o caso da cultivar ' 816 ', que apresentou baixo valor da massa da casca $(4,86 \mathrm{~g})$, mas com um boa massa de amêndoa $(3,20 \mathrm{~g})$.

Quanto à massa da "noz em casca", as cultivares 'Edson', 'Cannon', 'C160', 'Doroti' e '791
Fuji' se destacaram em relação às demais (Tabela 2). Porém, foram 'C160', 'HAES 849', 'IAC 4-12B', 'HAES 816' e 'Mauka' que apresentaram maior TR. Fato interessante foi observado para a cultivar 'Mauka', que apresentou elevado valor de TR, apesar do baixo valor das "nozes em casca". Isso se deve à massa da casca dessa cultivar, que foi apenas de 3,66g (Tabela 2). Ressalta-se que o fator determinante para um elevado TR não é somente a elevada massa fresca da amêndoa, mas também a baixa massa fresca da casca.

Apenas as cultivares 'Pahala', 'Beaumont', 'IAC 9-20X' e 'Keaumi' apresentaram TR menor que $30 \%$, ou seja, as demais cultivares possuem potencial para atingirem preços elevados na comercialização (PIMENTEL et al., 2007).

De acordo com os resultados obtidos, a cultivar 'Edson' apresentou frutos e nozes com maiores dimensões e massa. As cultivares 'C160', 'HAES 849', 'IAC 4-12B', 'HAES 816' e 'Mauka' apresentaram maior valor de TR. Apenas as cultivares 'Pahala', 'Beaumont', 'IAC 9-20X' e 'Keaumi' apresentaram TR menor que 30\%. 
Tabela 2 - Massa das partes dos frutos (carpelo, casca e amêndoa) e TR (relação entre a massa das nozes e a massa das amêndoas resgatadas) de 22 cultivares de nogueira-macadâmia cultivadas na região Leste paulista. Lavras, UFLA, 2011.

\begin{tabular}{|c|c|c|c|c|c|}
\hline \multirow{2}{*}{ Cultivares } & \multicolumn{4}{|c|}{--10 } & \multirow{2}{*}{$\mathrm{TR}(\%)$} \\
\hline & Carpelo & Casca & Amêndoa & Casca + Amêndoa ("noz em casca") & \\
\hline ‘Edson’ & 6,18 a & 6,80 a & 3,83 a & 10,64 a & $35,95 \mathrm{~b}$ \\
\hline 'Pahala (HAES 788)' & $2,89 \mathrm{f}$ & $5,50 \mathrm{~b}$ & $2,18 \mathrm{~b}$ & $7,73 \mathrm{~b}$ & $28,21 \mathrm{~d}$ \\
\hline 'Beaumont (695)’ & $3,05 \mathrm{e}$ & $4,87 \mathrm{c}$ & $1,65 \mathrm{~b}$ & $6,52 \mathrm{c}$ & $25,24 \mathrm{~d}$ \\
\hline 'Flor Rosa' & $2,11 \mathrm{~h}$ & $3,38 \mathrm{~d}$ & $1,87 \mathrm{~b}$ & $5,25 \mathrm{c}$ & $35,64 \mathrm{~b}$ \\
\hline 'IAC 9-20X’ & $2,98 \mathrm{f}$ & $5,18 \mathrm{c}$ & $2,06 \mathrm{~b}$ & $7,24 \mathrm{~b}$ & $28,47 \mathrm{~d}$ \\
\hline ‘Kau (HAES 344)’ & $2,90 \mathrm{f}$ & $5,45 \mathrm{~b}$ & $2,45 \mathrm{~b}$ & $7,90 \mathrm{~b}$ & $30,99 \mathrm{c}$ \\
\hline ‘Cannon’' & $3,65 \mathrm{~d}$ & 6,86 a & 3,06 a & 9,92 a & $31,13 \mathrm{c}$ \\
\hline 'IAC 9-20’' & $2,78 \mathrm{f}$ & $5,02 \mathrm{c}$ & $2,42 \mathrm{~b}$ & $7,45 \mathrm{~b}$ & $32,54 \mathrm{c}$ \\
\hline 'C160’' & $4,60 \mathrm{~b}$ & $5,34 \mathrm{~b}$ & 3,37 a & 8,71 a & 38,69 a \\
\hline 'HAES 849’ & $3,20 \mathrm{e}$ & $4,10 \mathrm{~d}$ & $2,49 \mathrm{~b}$ & $6,59 \mathrm{c}$ & 37,73 a \\
\hline 'IAC 4-12B' & $3,23 \mathrm{e}$ & $4,65 \mathrm{c}$ & $2,77 \mathrm{~b}$ & $7,42 \mathrm{~b}$ & 37,42 a \\
\hline 'HAES 816' & $3,43 \mathrm{~d}$ & $4,86 \mathrm{c}$ & $3,20 \mathrm{a}$ & $8,06 \mathrm{~b}$ & 39,70 a \\
\hline 'Doroti' & $3,52 \mathrm{~d}$ & $5,89 \mathrm{~b}$ & 3,29 a & 9,19 a & $34,85 \mathrm{~b}$ \\
\hline ‘791 Fuji’ & $2,83 \mathrm{f}$ & $5,71 \mathrm{~b}$ & 3,38 a & 9,59 a & $35,24 \mathrm{~b}$ \\
\hline 'Keaumi (IAC 4-20)’ & $3,16 \mathrm{e}$ & $5,03 \mathrm{c}$ & $1,95 \mathrm{~b}$ & $6,98 \mathrm{~b}$ & $27,94 \mathrm{~d}$ \\
\hline 'HAES 814' & $2,32 \mathrm{~g}$ & $4,37 \mathrm{~d}$ & $2,02 \mathrm{~b}$ & $6,39 \mathrm{c}$ & $31,65 \mathrm{c}$ \\
\hline 'HAES 722 & $3,53 \mathrm{~d}$ & $5,61 \mathrm{~b}$ & $2,46 \mathrm{~b}$ & $8,07 \mathrm{~b}$ & $30,47 \mathrm{c}$ \\
\hline ‘África’ & $4,26 \mathrm{c}$ & $5,56 \mathrm{~b}$ & $2,79 \mathrm{~b}$ & $8,35 \mathrm{~b}$ & $33,33 \mathrm{~b}$ \\
\hline 'IAC Campinas-B’' & $2,01 \mathrm{~h}$ & $4,27 \mathrm{~d}$ & $2,20 \mathrm{~b}$ & $6,49 \mathrm{c}$ & $34,24 \mathrm{~b}$ \\
\hline 'Keauhou (HAES 246)’ & $2,56 \mathrm{~g}$ & $5,02 \mathrm{c}$ & $2,16 \mathrm{~b}$ & $7,19 \mathrm{~b}$ & $30,06 \mathrm{c}$ \\
\hline ‘Mauka (HAES 741)’ & $2,01 \mathrm{~h}$ & $3,66 \mathrm{~d}$ & $2,30 \mathrm{~b}$ & $5,96 \mathrm{c}$ & 37,83 a \\
\hline 'HAES 842' & $2,31 \mathrm{~g}$ & $4,89 \mathrm{c}$ & $2,32 \mathrm{~b}$ & $7,21 \mathrm{~b}$ & $32,20 \mathrm{c}$ \\
\hline $\mathrm{CV}(\%)$ & 7,12 & 12,66 & 22,38 & 14,97 & 8,25 \\
\hline
\end{tabular}

*Médias não seguidas pela mesma letra diferem significativamente entre si, pelo teste Scott-Knott, em nível de 5\% de probabilidade.

\section{REFERÊNCIAS}

BARBOSA, W. et al. Distribuição geográfica e diversidade varietal de frutíferas e nozes de clima temperado no Estado de São Paulo. Revista Brasileira de Fruticultura, v.25, n.2, p.341-344, 2003. Disponível em: <http://www.scielo.br/scielo.php?script=sci_arttext\&pid=S0100 $29452003000200042 \& \operatorname{lng}=$ pt\&nrm=iso>. Acesso em: 27 abr. 2011. doi: $10.1590 / \mathrm{S} 0100-29452003000200042$.

CARVALHO, J.E.U. et al. Características físicas e físicoquímicas de um tipo de bacuri (Platonia insignis Mart.) com rendimento industrial superior. Revista Brasileira de Fruticultura, v.25, n.2, p.326-328, 2003. Disponível em: <http://www.scielo.br/scielo.php?script=sci_arttext\&pid=S010029452003000200036>. Acesso em: 27 abr. 2011. doi: 10.1590/ S0100-29452003000200036.

DALASTRA, I.M. Germinação de sementes de nogueira-macadâmia submetidas à incisão e imersão em ácido giberélico. Ciência e Agrotecnologia, v.34, n.3, p.641-645, 2010. Disponível em: <http://www.scielo.br/scielo.php?script=sci_arttext\&pid=S1413$70542010000300016 \& \operatorname{lng}=$ pt\&nrm=iso $>$. Acesso em: 28 abr. 2011. doi: $10.1590 / \mathrm{S} 1413-70542010000300016$
FERREIRA, D.F. Análise estatística por meio do SISVAR (Sistema para Análise de Variância) para Windows versão 4.0. In: REUNIÃO ANUAL DA REGIÃO BRASILEIRA DA SOCIEDADE INTERNACIONAL DE BIOMETRIA, 45., 2000, São Carlos. Anais... São Carlos: UFSCar, 2000. p.255258 .

GOMES, F.P. Curso de estatística experimental. 14.ed. Piracicaba: USP/ESALQ, 2000. 477p.

PEACE, C.P. et al. Genetic relationships amongst macadamia varieties grown in South Africa as assessed by RAF markers. South African Journal of Plant \& Soil, v.22, n.2, p.71-75, 2005 .

PIMENTEL, L.D. et al. Estudo de viabilidade econômica na cultura da noz-macadâmia no Brasil. Revista Brasileira de Fruticultura, v.29, n.3, p.500-507, 2007. Disponível em: $<$ http://www.scielo.br/scielo.php?script=sci_arttext\&pid=S0100$29452007000300018 \& \operatorname{lng}=$ pt \&nrm=iso >. Acesso em: 27 abr. 2011. doi: $10.1590 /$ S0100-29452007000300018.

SOBIERAJSKI, G.R. et al. Noz-macadâmia: produção, mercado e situação no Estado de São Paulo. Informações Econômicas, v.36, n.5, p.25-36, 2006. 